\title{
Excitonic structure and charge separation in the Heliobacterial Reaction Center probed by multispectral multidimensional spectroscopy
}

\author{
Yin Song ${ }^{1}$, Riley Sechrist ${ }^{1}$, Hoang H. Nguyen ${ }^{1}$, William Johnson ${ }^{2}$, Darius Abramavicius ${ }^{3}$, Kevin E. \\ Redding $^{2,4}$, and Jennifer P. Ogilvie ${ }^{1}$ \\ ${ }^{1}$ Department of Physics, University of Michigan, Ann Arbor, MI, 48109, US \\ ${ }^{2}$ School of Molecular Sciences, Arizona State University, Tempe, AZ, 85287, USA, \\ ${ }^{3}$ Institute of Chemical Physics, Faculty of Physics, Vilnius University, Sauletekio 9-III, 10222 Vilnius, \\ Lithuania \\ ${ }^{4}$ Center for Bioenergy and Photosynthesis, Arizona State University, Tempe, AZ, 85287, USA
}

\begin{abstract}
:
Photochemical reaction centers are the engines that drive photosynthesis. The reaction center from heliobacteria (HbRC) has been proposed to most closely resemble the common ancestor of photosynthetic reaction centers, motivating a detailed understanding of its structure-function relationship. The recent elucidation of the HbRC crystal structure motivates advanced spectroscopic studies of its excitonic structure and charge separation mechanism. We perform multispectral two-dimensional electronic spectroscopy of the HbRC and corresponding numerical simulations, resolving the electronic structure and testing and refining recent excitonic models. Through extensive examination of the kinetic data by lifetime density analysis and global target analysis, we reveal that charge separation proceeds via a single pathway in which the distinct $\mathrm{A}_{0}$ chlorophyll $a$ pigment is the primary electron acceptor. In addition, we find strong delocalization of the initial excited state and charge separation intermediate. Our findings have general
\end{abstract}


implications for the understanding of photosynthetic charge separation mechanisms, and how they might be tuned to achieve different functional goals. 


\section{Introduction}

Photosynthesis drives life on Earth by converting solar energy into chemical energy ${ }^{1,2}$. In photosynthesis, light is absorbed by the antenna pigments, which transfer their excitation energy to the reaction center $(\mathrm{RC})^{3,4}$. The primary energy conversion step, charge separation (CS), takes place in the $\mathrm{RC}^{5}$, driving the subsequent chemical reactions for synthesizing high-energy chemical compounds. All RCs are believed to have evolved from a common homodimeric ancestor $\mathrm{RC}^{6,7}$. Until recently, the crystal structures of the existing homodimeric RCs were unknown ${ }^{8}$, hindering mechanistic understanding of these systems. Recently, the crystal structure of the heliobacterial RC (HbRC) from Heliobacterium modesticaldum, was characterized by Gisriel and coworkers ${ }^{8}$. The HbRC has been proposed to be the RC most similar to the common ancestor of all photosynthetic RCs ${ }^{6,7}$. Furthermore, it is the simplest known RC and structural analog to the photosystem I RC ${ }^{9}$ (PSI RC), and possesses three chemically distinct pigments.

The HbRC binds 54 BChl $g$, four BChl $g^{\prime}$, two $8^{1}$-hydroxychlorophyll $a_{F}$ ( $8^{1}$-OH Chl $a$ ), two carotenoids (4,4'-diaponeurosporene), and one [4Fe-4S] cluster ${ }^{8}$. The majority of the BChl $g$ pigments serve as an antenna to direct excitation to the homodimeric core which serves as the electron transfer domain, depicted in Fig. 1. Throughout this paper we adopt the language commonly used in discussing RCs, where we reserve the term RC for the electron transfer domain, and the other pigments are collectively referred to as the "antenna”. The RC consists of two BChl $g$ ' pigments $\left(\mathrm{P}_{800}\right)$, two BChl $g$ (Acc), two Chl $a\left(\mathrm{~A}_{0}\right)$ and one [4Fe-4S] cluster ${ }^{8}$.

A structure-based excitonic model of the full HbRC was recently proposed by Kimura and coworkers $^{10}$. They report that the HbRC pigments form 58 delocalized excitonic states with 0-0 transition energies spanning 771 to $812 \mathrm{~nm}$ and two excitonic states with dominant absorption peaks centered at 667 nm. Until now, this model has not been tested by advanced experimental techniques.

The diverse pigment composition of the HbRC in comparison to other RCs makes it particularly appealing for spectroscopic studies of its primary CS processes. Energy transfer and CS in the HbRC have been studied by several groups ${ }^{11-21}$, with the early work reviewed by Neerken et al $^{17}$. In light of previous proposals of the CS mechanism and the new structure, Gisriel et al. proposed two possible 
charge-separation mechanisms: one in which Acc acts as the initial electron acceptor, and another in which Acc acts as the initial electron donor ${ }^{8}$.

Two-dimensional electronic spectroscopy (2DES) has proven to be a powerful tool to study photoexcited dynamics in a variety of systems including light-harvesting complexes ${ }^{22-24}$, photosynthetic $\mathrm{RCs}^{25,26}$ and solar cells ${ }^{27,28}$. A 2DES spectrum is a frequency-frequency map that directly highlights correlations between photoexcited and photodetected states via cross-peaks induced by excitonic interactions. 2DES also reveals the evolution of excited state populations, enabling the testing of kinetic models and the identification of spectroscopic fingerprints of reaction intermediates. Here were have applied 2DES to the HbRC, revealing excitonic correlations between Acc, $\mathrm{A}_{0}$ and $\mathrm{P}_{800}$ and refining the proposed excitonic structure. We find that CS in the HbRC proceeds via a two-step process in which $\mathrm{A}_{0}$ acts as the primary electron acceptor. Moreover, our results suggest that the hole is initially delocalized over Acc and $\mathrm{P}_{800}$ immediately after the primary CS event, but later becomes localized to $\mathrm{P}_{800}$. 


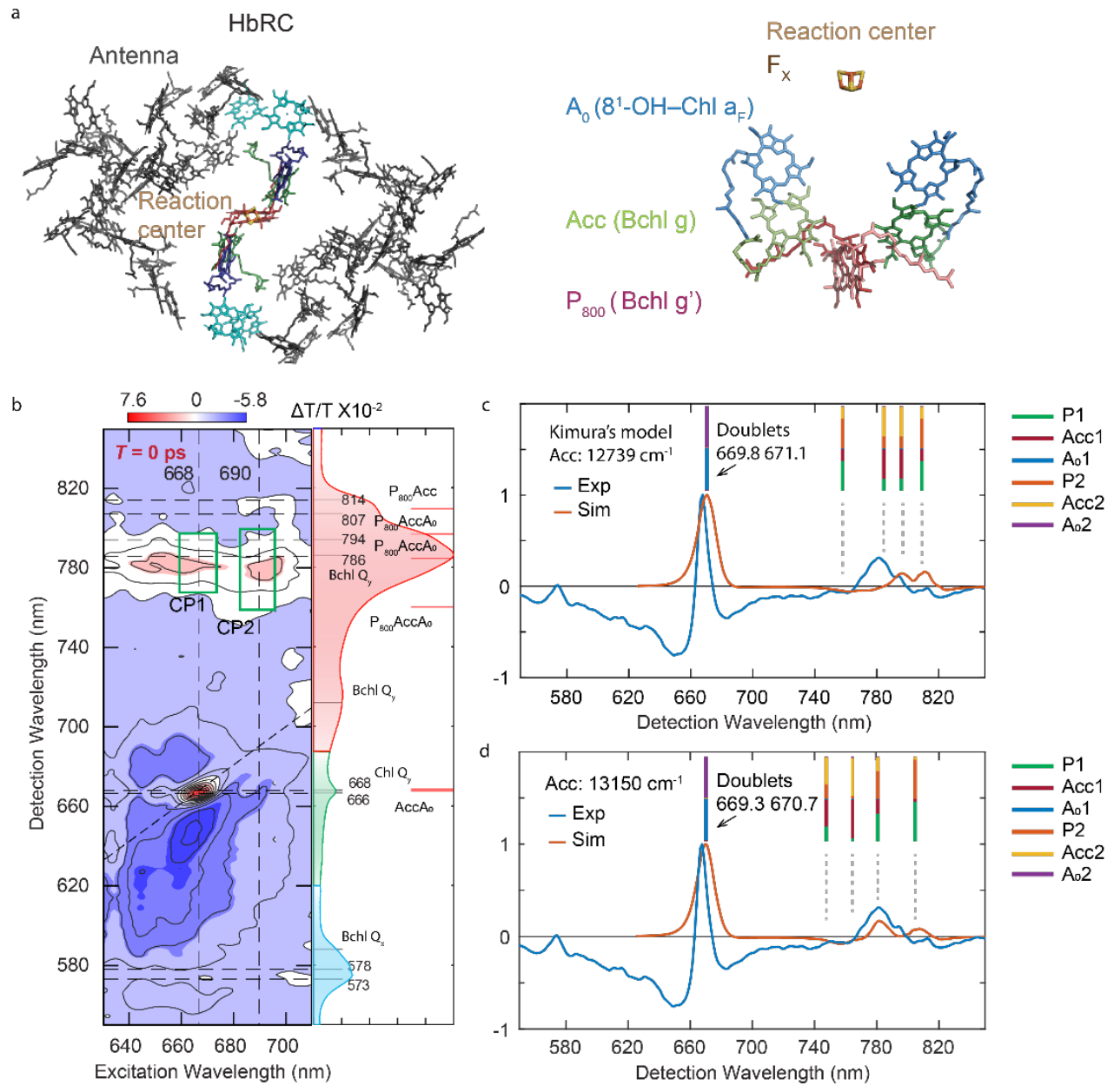

Fig. 1 Experimental and simulated 2DES absorptive spectra at $T=0$ ps reveal the excitonic structure of the core RC at 77K. a) The pigment arrangement in the full HbRC (left) and in the core RC (electron transfer domain). b) The experimental 2DES absorptive spectrum at $T=0 \mathrm{ps}$. Alongside the 2D absorptive spectra are the experimental absorption spectrum and the simulated stick spectrum (red lines) from Kimura et $\mathrm{al}^{10}$. The dashed lines in the 2DES spectrum and the grey lines in the absorption spectrum indicate the various peak positions discussed in the main text. c) The experimental and simulated slice spectra under excitation at $668 \mathrm{~nm}$ at $T=0 \mathrm{ps}$ extracted from the 2DES absorptive spectra. The site energies of BChl $g$ and BChl $g$ ' are set to be equal according to Kimura's excitonic model. d) The experimental and simulated slice spectra under excitation at $668 \mathrm{~nm}$ at $T=0$ ps extracted from the 2DES absorptive spectra. Modifying the site energy of accessory BChl $g$ to be $13150 \mathrm{~cm}^{-1}$ yields considerably better agreement with the experimental data. The presence of distinct BChl $\mathrm{Q}_{\mathrm{y}}$ peaks under excitation at $668 \mathrm{~nm}$ reveals excitonic delocalization between $\mathrm{A}_{0}$ and the BChl pigments Acc and $\mathrm{P}_{800}$. The stick bars reveal the participation ratios of the RC pigments in each excitonic state as given in the respective exciton models. 


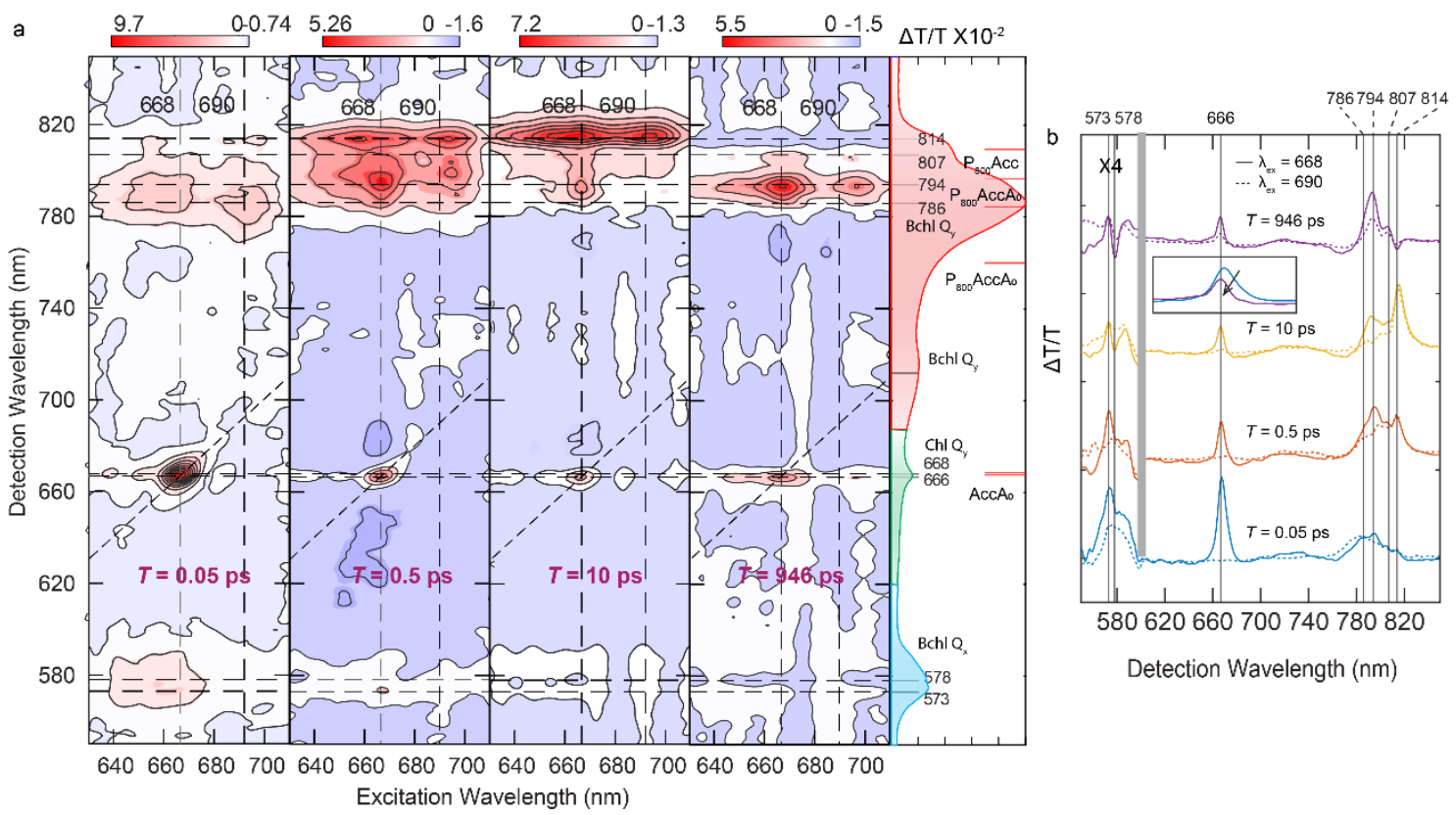

Fig. 2 2DES probes the photoexcited dynamics in the HbRC at 77K. a) 2DES absorptive spectra at 0.05 ps, 0.5 ps, $10 \mathrm{ps}$ and 946 ps. Alongside the 2D absorptive spectra are the experimental absorption spectrum and the simulated stick spectrum (red lines) from of Kimura et $\mathrm{al}^{10}$. The dashed lines in the 2DES and the grey lines in the absorption spectrum indicate the various peak positions discussed in the main text. b) The slice spectra under excitation at $668 \mathrm{~nm}$ (solid) and $690 \mathrm{~nm}$ (dashed) at various $T$ extracted from the 2DES absorptive spectra. The derivative lineshapes at 573, $578 \mathrm{~nm}$ and the blueshifted $\mathrm{A}_{0}$ peak at $666 \mathrm{~nm}$ (inset) are induced by the Stark effect due to the presence of an adjacent charge. These features are fingerprints for the charge-separated state. 


\section{Results}

To probe the HbRC excitonic structure and accentuate CS processes originating from RC excitation, we excited the $\sim 590-720 \mathrm{~nm}$ region, spanning the $\mathrm{Q}_{\mathrm{y}} \mathrm{A}_{0} \mathrm{Chl}$ and vibronic shoulder of $\mathrm{Q}_{\mathrm{y}} \mathrm{BChl}$ $g$ transitions. The multispectral probes in our recently developed 2DES instrument ${ }^{29}$ access both $\mathrm{Q}_{\mathrm{x}}$ and $\mathrm{Q}_{\mathrm{y}}$ of Chls and BChls, providing dynamical information about energy transfer and CS processes. We also performed complementary 2DES studies with excitation of the BChl $g$ Q $\mathrm{Q}_{\mathrm{y}}$ region.

Fig. 1b displays 2DES absorptive spectra of the HbRC at 77K, in which the sample was treated with sodium dithionite to reduce the terminal [4Fe-4S] cluster $\left(F_{x}\right)$. This blocks forward electron transfer from $\mathrm{A}_{0}$ to $\mathrm{F}_{\mathrm{x}}$, resulting in charge recombination of the $\mathrm{P}_{800} \mathrm{~A}_{0}^{-}$state in $\sim 15 \mathrm{~ns}^{12}$. The $2 \mathrm{D}$ absorptive spectrum at $T=0$ ps provides insight into the HbRC's excitonic structure and tests the current structurebased excitonic model ${ }^{10}$. The diagonal peak at $668 \mathrm{~nm}$ is consistent with photoexcitation of the highest energy RC excitons assigned to two states primarily involving $A_{0}$ with some participation from Acc. ${ }^{10}$ The presence of cross peaks in the region $\lambda_{\mathrm{ex}}=668 \mathrm{~nm}$ and $\lambda_{\text {det }}=770-800 \mathrm{~nm}$ (denoted CP1) is key to understanding the pigment composition of the excitonic states in the crowded BChl Qy region. The model of Kimura and coworkers ${ }^{10}$ predicts roughly three states in the $760-800 \mathrm{~nm}$ region that involve delocalization over $\mathrm{A}_{0}$ and could therefore produce $\mathrm{CP} 1$ in the $T=02 \mathrm{D}$ spectrum. These states, as well as the other RC excitons from the Kimura model, are indicated by solid red lines in the linear absorption spectrum shown in Fig. 1b (right panel). To test the Kimura exciton model, we simulated the 2DES absorptive spectrum in the CP1 region. The simulations employ a commonly modified Redfield approach, where the optical response functions are obtained within the adiabatic limit during electronic coherence periods: diagonal system-bath fluctuations are included exactly using the cumulant expansion, truncated at the second order, and off-diagonal fluctuations are included within Markovian second-order

perturbation theory ${ }^{30,31}$. The resulting slice spectrum upon excitation of $A_{0}$ is shown in Fig. 1c. The two cross peaks in the BChl $\mathrm{Q}_{\mathrm{y}}$ band provide firm evidence for excitonic delocalization, but are red shifted relative to the experimental data, indicating that refinement of the excitonic parameters is needed to capture the tuning of pigment site energies by the protein environment. We find better agreement between 
experiment and theory by shifting the site energy of Acc from $12739 \mathrm{~cm}^{-1}(785 \mathrm{~nm})$ to $13150 \mathrm{~cm}^{-1}(760 \mathrm{~nm}$ (see Fig. 1d). We also note that not all the excitonic peaks involving $A_{0}$ pigments appear in the simulated 2DES, due to low oscillator strength and overlapping contributions of the ground-state bleach (GSB), stimulated emission and excited state absorption (ESA) signals. Further simulation details are given in the Section S6 of Supplementary Information (SI).

While both the RC and the antenna excitons are simultaneously excited in this experiment, the spectral signatures are separated along the excitation and detection wavelengths. This enables investigation of the CS mechanism(s) initiated by direct excitation of the RC or excitation of the antenna. The 2DES spectra in Fig. 2 show that by 0.5 ps the 668-nm diagonal peak has largely decayed, accompanied by the rise of two cross peaks at $794 \mathrm{~nm}$ and $814 \mathrm{~nm}$. We interpret these signatures as redistribution of energy within the RC and equilibration with the antenna. The contribution of antenna excitons to these cross peaks is evident in the similarity of the cross-peak structure at an excitation wavelength of $690 \mathrm{~nm}$, which corresponds to predominantly antenna excitation. At 946 ps, the 2DES absorptive spectrum has become excitation wavelength independent, suggesting that excitons have been converted to the final chargeseparated state (CSS) - $\mathrm{P}_{800}{ }^{+} \mathrm{A}_{0}{ }^{-}$, as reported in previous studies ${ }^{11,14-18,32,33}$. In this 2D spectrum, a derivative lineshape near $575 \mathrm{~nm}\left(\mathrm{Q}_{\mathrm{x}}\right.$ band of BChl $\mathrm{g}$ ) shown in Fig. 2b is induced by the Stark effect due to the presence of an adjacent charge ${ }^{13}$, providing definitive fingerprints for CS. The CS is also evident in the evolution of the GSB peak of the 668-nm RC exciton, which shifts to $\lambda_{\text {det }}=666 \mathrm{~nm}$ in accordance with previous studies ${ }^{9,13}$, consistent with the formation of $A_{0}{ }^{-}$. Two other GSB peaks at $\lambda_{\text {det }}=794$ and $807 \mathrm{~nm}$ decay with $\mathrm{A}_{0}^{-}$and can thereby be assigned to the upper and the low excitonic peaks of $\mathrm{P}_{800}{ }^{+12,13}$.

To make full use of the rich information from 2DES, we used a combination of lifetime density analysis (LDA) $)^{34,35}$ to obtain an overview of the kinetics, followed by global-target analysis ${ }^{36}$ to test specific kinetic models. LDA fits the data using exponential functions with a continuous distribution of time constants. As no prior knowledge is required for this analysis, this approach is well suited to systems like the HbRC, in which multiple relaxation pathways are expected. Lifetime density maps (LDMs) that reveal the kinetics following RC and antenna excitation are shown in Supplementary Fig. 3 and 4 
respectively. LDM reveals the Stark lineshapes of the BChl $\mathrm{Q}_{x}$ peak (Supplementary Fig. 3b1) at 1.7 ps and 230 ps, which identify two time-windows for CS. At 1.7 ps, the Stark lineshape of the BChl $g$ Q $\mathrm{Q}_{\mathrm{x}}$ peak (Supplementary Fig. 3 panels b1-b3), does not yet exhibit the clear signatures of $\mathrm{P}_{800}{ }^{+} \mathrm{A}_{0}{ }^{-}$(i.e., peaks at $666 \mathrm{~nm}, 794 \mathrm{~nm}$ and $807 \mathrm{~nm}$ ), suggesting that an intermediate CSS exists on this timescale. The decay of the GSB of $A_{0}$ on this timescale is consistent with the participation of $A_{0}$ in the intermediate CSS and suggests that it is the primary electron acceptor. The LDMs for antenna excitation are consistent with this assignment, leading us to propose the following CS mechanism for the HbRC:

Upon direct excitation of the RC: $(\mathrm{RC})^{*} \rightarrow \operatorname{relaxed}(\mathrm{RC})^{*} \rightarrow$ intermediate $\mathrm{CSS} \rightarrow \mathrm{P}_{800}{ }^{+} \mathrm{A}_{0}{ }^{-}$

Upon antenna excitation: (Antenna exciton)* $\rightarrow$ relaxed (Antenna exciton) $\rightarrow\left(\right.$ RC) ${ }^{*} \rightarrow$ intermediate CSS $\rightarrow$ $\mathrm{P}_{800}{ }^{+} \mathrm{A}_{0}{ }^{-}$

To test the proposed CS mechanism and extract spectroscopic signatures of the intermediates, we turned to global-target analysis. This is often used in kinetic analysis of transient absorption spectroscopy $^{36}$ and has been applied in 2DES studies ${ }^{37-41}$. This approach fits the data using a trial kinetic model and produces a set of species-associated spectra (SAS) and rate constants. Guided by the LDA analysis and the revised Kimura model, we considered multiple kinetic models before arriving at the one presented in Fig. 3a. Further justification of our choice is given in Section S4 of the SI, where we discuss a subset of alternative models, including ones that incorporate additional and alternative CS pathways. The Fig. 3a model provides the best fit to the 2D data and produces SAS with spectroscopic characteristics consistent with the target model. In this model, two RC excitons and two antenna excitons are excited by our broadband pulses, after which downhill energy transfer precedes rapid CS via a single intermediate CSS. Species S0, with GSB peaks at $\lambda_{\text {det }}=668 \mathrm{~nm}$ and $780 \mathrm{~nm}$, and an ESA peak at $\lambda_{\text {det }}=$ $810 \mathrm{~nm}$, is assigned to the delocalized RC exciton (i.e., $\left.\left(\mathrm{A}_{0} \mathrm{Acc}\right)^{*}\right)$. This assignment is consistent with the presence of GSB peaks in the $T=0$ 2DES spectrum and is further supported by the large coupling strength of 200-300 $\mathrm{cm}^{-1}$ between $\mathrm{A}_{0}$ and $\mathrm{Acc}^{10,13}$ in the Kimura model. Our proposed shift of Acc's site 
energy suggests a stronger degree of participation from $\mathrm{P}_{800}$ in this $\mathrm{RC}$ exciton compared to the Kimura model.

After photoexcitation, S0 transitions to S1 with a time constant of 0.09 ps. S1 exhibits GSB peaks at $\lambda_{\text {det }}=668 \mathrm{~nm}$ and $790 \mathrm{~nm}$. S1 was not observed with excitation at $690 \mathrm{~nm}$ (see Supplementary Fig. 6), leading us to infer that S1 is also an RC excitonic state. Consistent with the Kimura model, we attribute S1 to a lower energy RC exciton involving some degree of delocalization over all RC pigments $\left(\mathrm{P}_{800} \mathrm{Acc}_{0}\right)^{* 10,42}$.

S1 transitions to S4 with a time constant of 0.89 ps. S4 exhibits three GSB peaks at $\lambda_{\text {det }}=666 \mathrm{~nm}, 794$ $\mathrm{nm}$ and $814 \mathrm{~nm}$ and a Stark lineshape near $\lambda_{\text {det }}=575 \mathrm{~nm}$. As discussed above, the presence of the Stark lineshape and the blue-shifted $\mathrm{A}_{0}$ peak are fingerprints of a CSS involving $\mathrm{A}_{0}$ and at least one BChl $g$ pigment. Compared to $\mathrm{P}_{800}{ }^{+} \mathrm{A}_{0}{ }^{-}$, S4 exhibits a larger splitting between the two GSB peaks in the BChl $\mathrm{Q}_{\mathrm{y}}$ band, suggesting the hole wavefunction in S4 may be more delocalized in this state than in $\mathrm{P}_{800}{ }^{+} \mathrm{A}_{0}$. Given the coupling strength of $\sim 200 \mathrm{~cm}^{-1}$ between Acc and $\mathrm{P}_{800}{ }^{10,42}$, we speculate that the hole wavefunction is delocalized over both of them in S4. One of the most striking features in this fit is that the CSS intermediate S4 is present in both charge-separation pathways, whether initiated by the antenna exciton or the RC exciton. To support this claim, we compared the fits of the pump-probe under excitation at $666 \mathrm{~nm}$ and $690 \mathrm{~nm}$ using one to three CS pathways (Supplementary Fig. 5-6) and the fits of the 2DES using two to three CS pathways (Supplementary Fig. 7-10). All of these fits show the presence of an intermediate CSS with the characteristics of S4, providing strong evidence that $A_{0}$ is the primary electron acceptor in the initial CS step, independent of the initial excitation. The intermediate CSS S4 relaxes to S5 with a time constant of 19 ps. S5 exhibits a derivative lineshape at $575 \mathrm{~nm}$, a blue-shifted $\mathrm{A}_{0}$ peak at $666 \mathrm{~nm}$ and two $\mathrm{P}_{800}{ }^{+}$peaks at 794 and $807 \mathrm{~nm}$ as described earlier, leading to its assignment as $\mathrm{P}_{800}{ }^{+} \mathrm{A}_{0}$.

We considered several models for the antenna relaxation, finding that a minimum of two distinct antenna excitons, S2 and S3, were needed. While S2 and S3 can be generated via direct photoexcitation of the vibronic shoulder of the $\mathrm{BChl} \mathrm{Q}_{\mathrm{y}}$ transitions, a small fraction of S0 is also transferred to the antenna, 
populating S2 and subsequently S3. We include a back-energy-transfer step from S3 to S2, inspired by a recent study ${ }^{43}$ that suggests the low-energy exciton is located far from the RC. The spectroscopic characteristics of S2 and S3 are consistent with their assignment as antenna excitons: both lack amplitude at $668 \mathrm{~nm}$ indicative of $\mathrm{A}_{0}$ excitation. S2 exhibits GSB signals at $\lambda_{\text {det }}=570-590 \mathrm{~nm}$ and $790-810 \mathrm{~nm}$ and transitions to the CSS S4 with a time constant of 2.2 ps. Due to the transfer of S0 to S2, we attribute S2 to an antenna exciton involving pigments near to the RC. In contrast, S3 exhibits GSB signals that are redshifted relative to S2 and undergoes relatively slow backwards energy transfer to S2, leading us to assign S3 to low-energy antenna exciton(s) ${ }^{16,18}$. We note that an RC exciton involving $\mathrm{P}_{800}$ and/or Acc only may be present between S2 and S4 and act as the reactant for the initial charge separation step. However, this component cannot be isolated from S2 probably due to their similar spectral profiles and fast exchange rate. 


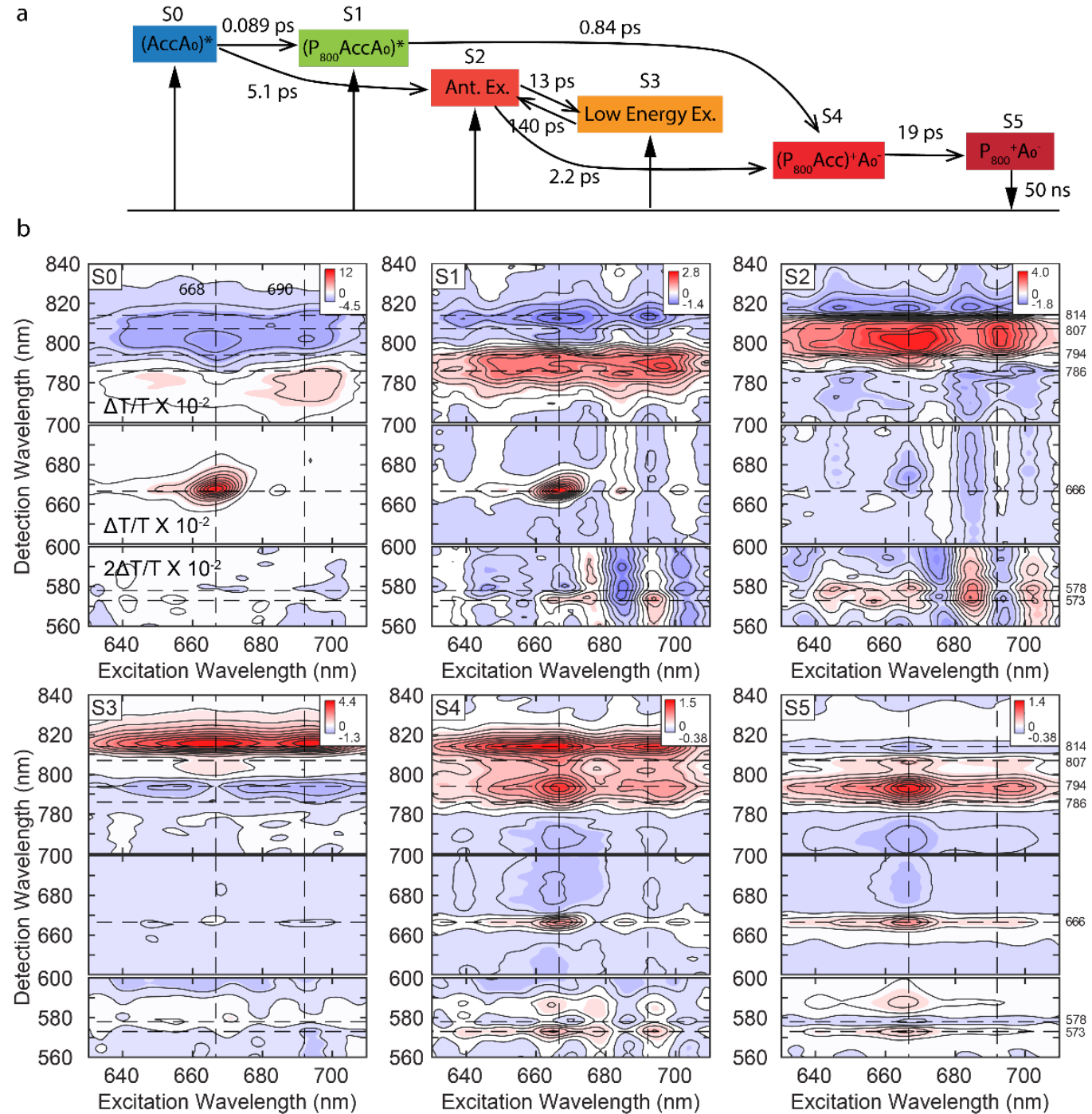

Fig. 3 | Target analysis of the HbRC reveals the kinetic map and insights into the participating states during CS. a) The kinetic model in which CS proceeds via a two-step mechanism with $\mathrm{A}_{0}$ as the primary electron acceptor, independent of whether the initial excitation occurs within the RC or antenna domains. The vertical arrows indicate species that can be generated by direct photoexcitation. b) The speciesassociated spectra of S0-S5 obtained from the global target analysis. The dashed lines in SASs indicate various peak positions as shown in Fig. 1-2 and discussed in the main text.

\section{Discussion}

The energy transfer and CS processes of the HbRC have been studied since the $1980 \mathrm{~s}^{11-18,20,21,44,45}$. Lin and coworkers ${ }^{11}$ reported that excitonic equilibrium within the antenna is complete within $\sim 0.8 \mathrm{ps,} \mathrm{while} \mathrm{CS}$ takes place at $\sim 25$ ps at room temperature. They further claimed that energy transfer is the rate-limiting step and reported the upper limit of the intrinsic CS to be $1 \mathrm{ps}^{-1}$. At cryogenic temperatures, Chiou et al. ${ }^{18}$ reported that all excitations are funneled into the lowest energy exciton prior to CS, which takes place 
within 55-70 ps. However, the $\operatorname{Vos}^{20}$ and Amesz groups ${ }^{16,17}$ observed fast CS occurring on the time scale of a few picoseconds upon photoexcitation of both antenna and $A_{0}$, suggesting that excitonic equilibrium need not be established prior to fast CS.

Another debate concerns the difference in CS mechanisms upon excitation of the RC and antenna domains ${ }^{16-18}$. Initially, a one-step CS mechanism (i.e., hole transfer from $\mathrm{A}_{0}$ to $\mathrm{P}_{800}$ or electron transfer from $\mathrm{P}_{800}$ to $\mathrm{A}_{0}$ ) was used to describe CS for both RC and antenna excitation ${ }^{16,17,20,44}$. However, the onestep mechanism cannot explain the multiple time scales observed for CS, or 'uphill' CS from the lowest energy antenna exciton ${ }^{16,18,21}$. To resolve these issues, Chiou et $\mathrm{al}^{18}$ proposed a two-step mechanism in which $A_{0}$ is the primary acceptor. Without knowledge of the location of $A_{0}$ and its coupling to other pigments, the feasibility of this mechanism, and the relationship to CS mechanisms in other RCs, were unclear.

Upon resolution of the HbRC structure, Gisriel et al. ${ }^{8}$ proposed two different two-step CS mechanisms for the HbRC. In the first, Acc acts as the primary electron acceptor, similar to the dominant mechanism proposed for the purple bacterial $\mathrm{RC}^{37,46}$. In the second, Acc acts as the primary electron donor, with $\mathrm{A}_{0}$ playing the role of primary electron acceptor in analogy to $\operatorname{PSI}^{1}$ and the dominant mechanism in both PSI $^{35,47-49}$ and PSII $^{35,49,50}$. The LDA and global target analysis of our 2DES data strongly favor the latter mechanism. Regardless of initial excitation, RC and antenna excitons both transition to a common intermediate CSS (S4), in which $\mathrm{A}_{0}$ is the primary electron acceptor and the hole wavefunction is delocalized over Acc and $\mathrm{P}_{800}$. We cannot entirely rule out additional CS intermediates obscured by spectral congestion in the BChl $\mathrm{Q}_{\mathrm{y}}$ region and overlapping time scales of energy equilibration and primary CS. However, our extensive consideration of other target models consistently found that any intermediates exhibiting the hallmark of a CSS also involved $\mathrm{A}_{0}$.

The mechanism we have proposed makes good chemical and biological sense. We have argued that the ancestral homodimeric RC used quinones as terminal electron acceptors to couple light-driven electron transfer to proton pumping across the membrane, driving ATP production. Inherent inefficiencies of quinone reduction in this RC drove the evolution of type I and type II RCs ${ }^{9}$. While type II RCs became 
heterodimeric and specialized the two branches, type I RCs added the $\mathrm{F}_{\mathrm{X}}$ cluster to catalyze semiquinone dismutation; the subsequent switch to reducing ferredoxin necessitated a more reducing cluster as well as an upstream donor $\left(A_{0}\right)$ with much lower reduction potential. This explains why all type I RCs use a version of $\mathrm{Chl} a$ in the $\mathrm{A}_{0}$ site due to the considerably lower reduction potentials of chlorins, including $\mathrm{A}_{0}{ }^{12}$, compared to bacteriochlorins ${ }^{51,52}$. The use of $\mathrm{Chl} a$ as $\mathrm{A}_{0}$ places serious constraints on the CS mechanism. It would be extremely difficult for $\mathrm{Acc}^{-}$(BChl) to act as an electron donor to $\mathrm{A}_{0}$ (Chl), rendering the "purple bacterial RC model" very unlikely. This means that the excited state of BChl $g$, which has a significantly lower potential than the ground state, must be used as the primary donor to $\mathrm{A}_{0}$. Our data are most consistent with a model in which the primary donor is actually an excited state delocalized across several BChl $g$ ( $\mathrm{P}_{800}$ and Acc). This mechanism may protect the HbRC from wasting energy through charge recombination reactions: delocalization of the hole moves cation density away from $\mathrm{A}_{0}{ }^{-}$in the initial CSS, which should serve to slow charge recombination of the initial radical ion pair. This may be especially important for anoxygenic type I RCs, in which the redox gradient from BChl to Chl would be uphill if not for the use of the excited state. (In contrast, the redox gradient from (B)Chl to (B)Pheo is downhill in type II RCs.) The subsequent step of localizing the hole to the $\mathrm{P}_{800} \mathrm{BChl}$ dimer would be a natural consequence of its higher reduction potential, enabling the special pair to stabilize the hole and place it near the external side for reduction by the external donor (cytochrome $c$ ).

In conclusion, multispectral 2DES has provided insight into the excitonic structure and CS mechanism of the HbRC. Our extensive kinetic analysis revealed $A_{0}$ as the primary electron acceptor independent of whether the initial excitation occurs in the RC or antenna domain. We find RC excitonic delocalization over Acc and $A_{0}$ while the intermediate CSS is delocalized over $P_{800}$, Acc and $A_{0}$. The use of delocalization across the special pair and accessory pigments could well be a general strategy used by other photochemical $\mathrm{RCs}^{53}$. The HbRC presents an especially tractable case, due to the chemically different pigment in the $\mathrm{A}_{0}$ site. Our study highlights the different ways in which nature has made use of the basic RC architecture that emerged over 3 billion years ago. 


\section{Methods}

Sample Preparation: The isolation of the HbRC is described in previous studies ${ }^{13}$. The HbRC sample was stored in a buffer solution that contains $5.38 \mu \mathrm{M}$ HbRC, $100 \mathrm{mM}$ glycine buffer $(\mathrm{pH}=10), 0.02 \% \mathrm{n}$ dodecyl- $\beta$-D-maltoside. Before ultrafast spectroscopic measurements, the HbRC solution was treated with $20 \mathrm{mM}$ dithionite to reduce the terminal [4Fe-4S] cluster $\left(\mathrm{F}_{\mathrm{x}}\right)$. This blocks forward electron transfer from $\mathrm{A}_{0}$ to $\mathrm{F}_{\mathrm{x}}$, resulting in charge recombination of the $\mathrm{P}_{800} \mathrm{~A}_{0}{ }^{-}$state in $\sim 15 \mathrm{~ns}^{12}$. The 1:1 mixture of the HbRC solution and glycerol was loaded into a sealed quartz sample cell with an optical path length of $380 \mu \mathrm{m}$. The OD values at $786 \mathrm{~nm}$ and at $668 \mathrm{~nm}$ of the samples were 1.1 and 0.13 , respectively. The transient measurements were performed at $74 \mathrm{~K}$ using a liquid nitrogen cryostat from Oxford Instrument.

Spectroscopic Measurements: 2DES spectra were measured by using a pump-probe geometry 2DES setup as described previously (see Section S5 of SI for further details). ${ }^{29}$. The pump pulse was compressed to 12 fs. A two-phase cycling scheme was used to remove scattering and background signals. A shutter added in the probe arm removed residual scattering from the pump. In the experiments, the pulse energy of pump pulses was $\sim 40 \mathrm{~nJ}$ and the beam waists $\left(1 / \mathrm{e}^{2}\right)$ for both pump and probe were $\sim 200 \mu \mathrm{m}$. 2D experiments were performed under the magic-angle condition and at least three times to ensure reproducibility. The data was analyzed using home-written MatLab scripts. The chirp of the probe pulse was corrected using a third-order polynomial function as described previously ${ }^{54}$. The lifetime density analysis was performed using the OPTIMUS software ${ }^{34}$. The global-target analysis was implemented using CarpetView3D (Light Conversion). Fluence dependence studies were also conducted to avoid the exciton-exciton, exciton-charge, and charge-charge annihilation (Supplementary Fig. 12).

\section{Acknowledgements}

Y.S., R. S., H. N. and J.P.O. acknowledge support from the Office of Basic Energy Sciences, the U.S. Department of Energy, under Grant Number DE-SC0016384. DA acknowledges support of the Lithuanian Science Council (grant No. S-MIP-20-47). 


\section{Author contributions}

Y.S., K.E.R. and J.P.O. conceived and designed the experiments. Y.S. and R.S. performed the experiments. Y.S. analyzed the data. H.H.N. and D.A. performed simulations. W.J. isolated the HbRC proteins and prepared the samples. Y.S., J.P.O., K.E.R. and D.A. wrote the manuscript, with input from all the authors.

\section{Additional information}

Supplementary information is available in the online version of the paper. Reprints and permissions information is available online at www.nature.com/reprints. Correspondence and requests for materials should be addressed to J.P.O.

\section{Competing interests}

The authors declare no competing financial interests.

\section{References}

1 Savikhin, S. in Photosystem I: The Light-Driven Plastocyanin:Ferredoxin Oxidoreductase (ed John H. Golbeck) 155-175 (Springer Netherlands, 2006).

2 Blankenship, R. E. in Molecular Mechanisms of Photosynthesis Wiley Online Books 1-10 (Blackwell Science Ltd, 2002).

3 Blankenship, R. E. in Molecular Mechanisms of Photosynthesis Wiley Online Books 61-94 (Blackwell Science Ltd, 2002).

4 Mirkovic, T. et al. Light Absorption and Energy Transfer in the Antenna Complexes of Photosynthetic Organisms. Chemical Reviews 117, 249-293, doi:10.1021/acs.chemrev.6b00002 (2017).

5 Blankenship, R. E. in Molecular Mechanisms of Photosynthesis Wiley Online Books 124-156 (Blackwell Science Ltd, 2002).

6 Khadka, B., Adeolu, M., Blankenship, R. E. \& Gupta, R. S. Novel insights into the origin and diversification of photosynthesis based on analyses of conserved indels in the core reaction center proteins.

$7 \quad$ Olson, J. M. \& Blankenship, R. E. Thinking About the Evolution of Photosynthesis. Photosynthesis Research 80, 373-386, doi:10.1023/B:PRES.0000030457.06495.83 (2004).

8 Gisriel, C. et al. Structure of a symmetric photosynthetic reaction center-photosystem. Science 357, 1021-1025, doi:10.1126/science.aan5611 (2017)

9 Orf, G. S., Gisriel, C. \& Redding, K. E. Evolution of photosynthetic reaction centers: insights from the structure of the heliobacterial reaction center. Photosynth Res 138, 11-37, doi:10.1007/s11120-018-0503-2 (2018).

10 Kimura, A. \& Itoh, S. Theoretical Model of Exciton States and Ultrafast Energy Transfer in Heliobacterial Type I Homodimeric Reaction Center. J Phys Chem B 122, 11852-11859, doi:10.1021/acs.jpcb.8b08014 (2018).

11 Lin, S., Chiou, H. C., Kleinherenbrink, F. A. \& Blankenship, R. E. Time-resolved spectroscopy of energy and electron transfer processes in the photosynthetic bacterium Heliobacillus mobilis. Biophysical Journal 66, 437-445, doi:10.1016/S00063495(94)80794-5 (1994).

12 Ferlez, B. et al. Thermodynamics of the Electron Acceptors in Heliobacterium modesticaldum: An Exemplar of an Early Homodimeric Type I Photosynthetic Reaction Center. Biochemistry 55, 2358-2370, doi:10.1021/acs.biochem.5b01320 (2016).

13 Chauvet, A. et al. Temporal and spectral characterization of the photosynthetic reaction center from Heliobacterium modesticaldum. Photosynth Res 116, 1-9, doi:10.1007/s11120-013-9871-9 (2013).

14 Nuijs, A. M., Dorssen, R. J. v., Duysens, L. N. M. \& Amesz, J. Excited States and Primary Photochemical Reactions in the Photosynthetic Bacterium Heliobacterium Chlorum. Proceedings of the National Academy of Sciences 82, 6865, doi:10.1073/pnas.82.20.6865 (1985).

15 Vannoort, P. I., Gormin, D. A., Aartsma, T. J. \& Amesz, J. Energy-Transfer and Primary Charge Separation in HeliobacteriumChlorum Studied by Picosecond Time-Resolved Transient Absorption-Spectroscopy. Biochim Biophys Acta 1140, 15-21, doi:Doi 10.1016/0005-2728(92)90014-S (1992).

16 Neerken, S., Aartsma, T. J. \& Amesz, J. Pathways of Energy Transformation in Antenna Reaction Center Complexes of Heliobacillus mobilis. Biochemistry 39, 3297-3303, doi:10.1021/bi992433o (2000).

17 Neerken, S. \& Amesz, J. The antenna reaction center complex of heliobacteria: composition, energy conversion and electron transfer. Bba-Bioenergetics 1507, 278-290, doi:Doi 10.1016/S0005-2728(01)00207-9 (2001). 
Chiou, H.-C., Lin, S. \& Blankenship, R. E. Time-Resolved Spectroscopy of Energy Transfer and Trapping upon Selective Excitation in Membranes of Heliobacillus mobilis at Low Temperature. The Journal of Physical Chemistry B 101, 4136-4141, doi:10.1021/jp963384h (1997).

Allodi, M. A. et al. Redox Conditions Affect Ultrafast Exciton Transport in Photosynthetic Pigment-Protein Complexes. J Phys Chem Lett 9, 89-95, doi:10.1021/acs.jpclett.7b02883 (2018).

Liebl, U. et al. Energy and Electron Transfer upon Selective Femtosecond Excitation of Pigments in Membranes of Heliobacillus mobilis. Biochemistry 35, 9925-9934, doi:10.1021/bi960462i (1996).

Kojima, R. et al. Energy transfer and primary charge separation upon selective femtosecond excitation at $810 \mathrm{~nm}$ in the reaction center complex from Heliobacterium modesticaldum. Journal of Photochemistry and Photobiology A: Chemistry 401, 112758, doi:https://doi.org/10.1016/j.jphotochem.2020.112758 (2020).

Engel, G. S. et al. Evidence for wavelike energy transfer through quantum coherence in photosynthetic systems. Nature 446, 782-786, doi:10.1038/nature05678 (2007).

Paleček, D., Edlund, P., Westenhoff, S. \& Zigmantas, D. Quantum coherence as a witness of vibronically hot energy transfer in bacterial reaction center. Science Advances 3, e1603141, doi:10.1126/sciadv.1603141 (2017).

Collini, E. et al. Coherently wired light-harvesting in photosynthetic marine algae at ambient temperature. Nature 463, 644-647, doi:10.1038/nature08811 (2010).

Romero, E. et al. Quantum coherence in photosynthesis for efficient solar-energy conversion. Nature Physics 10, 676-682, doi:10.1038/nphys3017 (2014).

Fuller, F. D. et al. Vibronic coherence in oxygenic photosynthesis. Nature Chemistry 6, 706-711, doi:10.1038/nchem.2005 (2014). Song, Y., Clafton, S. N., Pensack, R. D., Kee, T. W. \& Scholes, G. D. Vibrational coherence probes the mechanism of ultrafast electron transfer in polymer-fullerene blends. Nat Commun 5, doi:10.1038/ncomms5933 (2014).

De Sio, A. et al. Tracking the coherent generation of polaron pairs in conjugated polymers. Nat Commun 7, 13742, doi:10.1038/ncomms13742 (2016).

Song, Y. et al. Multispectral multidimensional spectrometer spanning the ultraviolet to the mid-infrared. Rev Sci Instrum 90, doi:10.1063/1.5055244 (2019). Excitons in Molecular Aggregates; Quasiparticle versus Supermolecule Perspectives. Chemical Reviews 109, 2350-2408, doi:10.1021/cr800268n (2009).

Abramavicius, D., Valkunas, L. \& Mukamel, S. Transport and correlated fluctuations in the nonlinear optical response of excitons. Epl-Europhys Lett 80, doi:10.1209/0295-5075/80/17005 (2007).

Amesz, J. The heliobacteria, a new group of photosynthetic bacteria. Journal of Photochemistry and Photobiology B: Biology 30, 8996, doi:https://doi.org/10.1016/1011-1344(95)07207-I (1995).

Hastings, G., Hoshina, S., Webber, A. N. \& Blankenship, R. E. Universality of Energy and Electron Transfer Processes in Photosystem I. Biochemistry 34, 15512-15522, doi:10.1021/bi00047a017 (1995).

Slavov, C., Hartmann, H. \& Wachtveitl, J. Implementation and evaluation of data analysis strategies for time-resolved optical spectroscopy. Anal Chem 87, 2328-2336, doi:10.1021/ac504348h (2015).

Holzwarth, A. R. et al. Kinetics and mechanism of electron transfer in intact photosystem II and in the isolated reaction center: Pheophytin is the primary electron acceptor. Proceedings of the National Academy of Sciences 103, 6895, doi:10.1073/pnas.0505371103 (2006).

van Stokkum, I. H. M., Larsen, D. S. \& van Grondelle, R. Global and target analysis of time-resolved spectra. Biochimica et Biophysica Acta (BBA) - Bioenergetics 1657, 82-104, doi:https://doi.org/10.1016/j.bbabio.2004.04.011 (2004).

Niedringhaus, A. et al. Primary processes in the bacterial reaction center probed by two-dimensional electronic spectroscopy. Proceedings of the National Academy of Sciences of the United States of America 115, 3563-3568, doi:10.1073/pnas.1721927115 (2018).

Lewis, K. L. M. \& Ogilvie, J. P. Probing Photosynthetic Energy and Charge Transfer with Two-Dimensional Electronic Spectroscopy. The Journal of Physical Chemistry Letters 3, 503-510, doi:10.1021/jz201592v (2012).

Milota, F. et al. Vibronic and Vibrational Coherences in Two-Dimensional Electronic Spectra of Supramolecular J-Aggregates. J Phys Chem A 117, 6007-6014, doi:10.1021/jp3119605 (2013).

Ostroumov, E. E., Mulvaney, R. M., Cogdell, R. J. \& Scholes, G. D. Broadband 2D Electronic Spectroscopy Reveals a Carotenoid Dark State in Purple Bacteria. Science 340, 52-56, doi:10.1126/science.1230106 (2013).

Lee, Y., Gorka, M., Golbeck, J. H. \& Anna, J. M. Ultrafast Energy Transfer Involving the Red Chlorophylls of Cyanobacterial Photosystem I Probed through Two-Dimensional Electronic Spectroscopy. Journal of the American Chemical Society 140, 1163111638, doi:10.1021/jacs.8b04593 (2018).

Kitoh-Nishioka, H., Shigeta, Y., Itoh, S. \& Kimura, A. Excitonic Coupling on a Heliobacterial Symmetrical Type-I Reaction Center: Comparison with Photosystem I. J Phys Chem B 124, 389-403, doi:10.1021/acs.jpcb.9b11290 (2020).

Orf, G. S., Gisriel, C., Baker, P. \& Redding, K. E. The PshX subunit of the photochemical reaction center from Heliobacterium modesticaldum acts as a low-energy antenna. Unpublished.

Liebl, U., Lambry, J. C., Breton, J., Martin, J. L. \& Vos, M. H. Spectral equilibration and primary photochemistry in Heliobacillus mobilis at cryogenic temperature. Biochemistry 36, 5912-5920, doi:DOI 10.1021/bi9625197 (1997).

Redding, K. E. et al. Modulation of the fluorescence yield in heliobacterial cells by induction of charge recombination in the photosynthetic reaction center. Photosynth Res 120, 221-235, doi:10.1007/s11120-013-9957-4 (2014).

Zinth, W. \& Wachtveitl, J. The first picoseconds in bacterial photosynthesis - Ultrafast electron transfer for the efficient conversion of light energy. Chemphyschem 6, 871-880, doi:10.1002/cphc.200400458 (2005).

Prokhorenko, V. \& Holzwarth, A. R. Primary charge separation at low temperatures in D1-D2 reaction centers studied by photon echo and pump-probe spectroscopy. (1998). Pathways in Photosystem II. Biochemistry 49, 4300-4307, doi:10.1021/bi1003926 (2010).

Groot, M. L. et al. Initial electron donor and acceptor in isolated Photosystem II reaction centers identified with femtosecond mid-IR spectroscopy. Proceedings of the National Academy of Sciences of the United States of America 102, 13087, doi:10.1073/pnas.0503483102 (2005). 
Prokhorenko, V. I. \& Holzwarth, A. R. Primary processes and structure of the photosystem II reaction center: A photon echo study. Journal of Physical Chemistry B 104, 11563-11578, doi:10.1021/jp002323n (2000).

51 Watanabe, T. \& Kobayashi, M. in Chlorophylls (ed Hugo Scheer) Ch. Electrochemistry of Chlorophylls, 287-303 (CRC Press, Boca Raton, FL, 1991).

52 Fajer, J. et al. in Antennas and Reaction Centers of Photosynthetic Bacteria (ed Maria Elisabeth Michel-Beyerle) Ch. Experimental, structural and theoretical models of bacteriochlorophylls a, d, and g, 324 (Springer-Verlag, Berlin, 1985).

53 Abramavicius, D. \& Mukamel, S. Exciton Delocalization and Transport in Photosystem I of Cyanobacteria Synechococcus elongates: Simulation Study of Coherent Two-Dimensional Optical Signals. Journal of Physical Chemistry B 113, 6097-6108,

doi:10.1021/jp811339p (2009).

54 Tekavec, P. F., Myers, J. A., Lewis, K. L. M., Fuller, F. D. \& Ogilvie, J. P. Effects of chirp on two-dimensional Fourier transform electronic spectra. Opt Express 18, 11015-11024, doi:10.1364/Oe.18.011015 (2010). 